\section{No Time to Think A Theory about What Architects Do in the Age of Artificial Intelligence [AI]}

Shai Yeshayahu

Ryerson University

Eric Strian

University of Las Vegas Nevada

\section{Maria Vera}

University of Las Vegas Nevada

\section{INTRODUCTION}

History tells us that the nine-square grid did not ignite the education of an architect, ${ }^{1}$ blobitecture did not stifle it, ${ }^{2}$ and DIY software is not killing the profession. ${ }^{3}$ Instead, the duration of time allotted to aggregate knowledge and implement research in both learning and practice is under attack. At risk is the logic for how humans cede cognitive praxes to machines. ${ }^{4}$

In other words, for space thinkers and designers, the time to output results is vastly shrinking, challenging the ways we teach, learn, and gain the ability to apply innovative research outputs mindfully. Should having no time to evaluate and assimilate the particularities of our cognitive experiences in meaningful ways worry us? The answer is an emphatic Yes! At the crux of this response lies the claim that Artificial Intelligence [AI] and deep learning are singularly computational systems capable of evolutionary acts and random mutations that will continuously deliver optimal answers upon request. ${ }^{5}$

How and in what ways has Architecture (AIA 2018) concede that design development, construction documents, and building construction are a mundane task, left for machines to execute in the absence of design innovators? If so, what are the tasks of architects, and how will innovation and creative-thinking continue to evolve beyond Al?

\section{TASK}

Within a plethora of discoveries and research-work, immersed in experimentation, conversations, exhibits, books, buildings, laws, films, and more lies the evidence for how humans have conditioned realities over time. Increasingly, however, machine learning enthusiasts believe that design operations are like any data: a type of retrieval process based on rules, numbers, and patterns, absent of contextual meaning. Rattled by these diverging accounts of future design practices our team sought a collaborative investigation to pursue means that re-explore learning as a craft based on time to meditatively inform their ideas and means to calibrate their design sensitivities. The goal is to teach in ways that will allow students to learn and grasp the magnitude of the design problem and to address its complexity based on experiential research, deductive reasoning, and crafted work. We seek to strengthen the means for exploring ideas, driven by the knowledge and tools acquired through thoughtful systems of education that empowers their trust in pursuing design approaches beyond intuitive hunches.

Energized by all kinds of possible paths, our teaching positions stepped away from the increasing prerequisites that NAAB, IDEC, and $L A A B$ superimposed on standardized curricula to focus on the strategies that foster design learning as a skilled trade. We were less concerned with programmatic content and more interested in the time students allocate for deep learning. Throughout the semester, we emphasized the interplay of slow learning using practice-based methods attributed to artists, artisans, and craftsmen. 


\section{INSPIRATION}

"It's that bloody filthy Paris wine and the filthy fat of the steaks that do that to you - dear God, I had come to a state in which my own

blood was no longer working at all." 6

From Vincent Van Gogh's denunciation of Paris as an overflow of distraction and consumption, we identified our data-rich world as the new filthy environment, poisoning the creativity out of our systems. Then, looking for discourse among scholars working through pedagogic platforms, we made efforts to avoid personal ideological positions that often curtail engagement in addressing the teaching polemics of our times.

Our team, three faculty members, two teaching design-based studios, and the third teaching a seminar-based class, elucidated the means to identify logics and tools that would foster, expand, and protect abstract reasoning. Based on the purview of three eminent historians and one cognitive psychologist, we each grappled independently with the changing characteristics of our learning milieu, each of us identified ways to develop syllabus and teaching strategies that would aid our intent. This collaborative approach illustrates the methods for how we tinkered with time to improve student's thinking skills and nurture their meditative approach for crafting design logics. Thus, what follows is the rationale unfolding across a semester-long exploration for "time to think." It is a depiction of three ways to craft learning in slow motion, as it becomes fundamentally clear that algorithms shuffle, process, sort, and classify data faster than humans ever will.

\section{PRELUDE}

\section{THREE HISTORIANS}

I. The Radical Pedagogies Project ${ }^{7}$ [RPP]

led by Beatriz Colomina lends credence to a diverse network of global research on the ongoing debate about the education of an architect post-WWII. In part, this project continues to educate educators about pedagogic instances where "subversion took multiple forms: challenging programs, architecture schools, institutions and even the basic relationship between teacher and students." 8

The website, alone, works as a reshuffling system, enabling academics to perpetually reconstruct the education of architects and continue to evolve beyond the evidence of the actual production from the last century. Within the bounds of the upcoming book, previous lectures, and exhibits, one can also assimilate ideas set forth by authors and curators who are provoking us with insightful reflections about design and teaching. At the 14th Venice Biennale of Architecture (Jun 7Nov 23, 2014) the RPP team call for the Action-Reaction-Interaction of education by exhibiting "a series of pedagogical experiments that played a crucial role in shaping architectural discourse and practice in the second half of the twentieth century." 9 Educators flocking to Venice took notes, listened to lectures and conversations making the title of this exhibit an internalized call igniting new kinds of educational narratives. "The exhibition was awarded a Special Mention, cited by the jury for "highlight[ing] the emergence of new poles of architectural thinking in the current world and mak[ing] these accessible as a living archive." 10

As their research work continues, the message sets a stage for reflection on the state of education and design as a collective of multiple intelligences. It is becoming a tool to implement teaching and learning beyond institutional prerogative.

II. In The Second Digital Turn: Design Beyond Intelligence, ${ }^{11}$ Mario Carpo writes about the task of architects across past centuries, during the twentieth century, at the digital turn of 1992-2012, and now as the start of the second digital turn unfolds. Carpo explicitly postulates that from the Renaissance times until the end of the twentieth century, the education of an architect was based on notations, exclusively created to instruct builders how to build. His view of the second digital turn highlights the transformation of how and why we currently notate. The trajectory of building, according to Carpo, has once again veered architects to re-develop expertise similar to those of ancient artisans, where specialized skills and knowledge augment with time and practice as artisans ideate, tweak forms, and built directly with the material's logic. To grasp this shift, Carpo explains how, in the absence of builders, fabricators, or engineers, designers adapt and make new outcomes on command. This narration is key to understanding the historical journey of Al, which, according to Carpo, will inevitably take over the logic of how we design. From his research work, Carpo tells students that focusing on school's prerequisites will not suffice for the near future. He tells learners "when you are in a studio, you have urgent things to do, and there is not the time to stop, take stock, and think, because everything is driven by the design assignment you are given. Now, we think, in some cases it may be worth taking some time off, say a few months; you read some books, meet some people, listen to a few lectures, write a paper, and then, if you are a designer, you may go back to design but when you do, you will be a better designer." 12

Through his lectures, the pathology of design requires designers to understand material logic as ancient artisans did, the act of inputting notations is an encompassing approach that leads directly to fabrication, which in turn has its roots in the art of design thinking.

III. Architectural Intelligence, a book by Molly Wright Steenson, narrates the "architectural history of digital design and a digi-tal history of architecture, with deep case studies on the work of Christopher Alexander, Richard Saul Wurman, Nicholas Negroponte, and Cedric Price, and the ways that their work influenced the development of contemporary digital design practices, including information architecture and interaction design." 13

Humans have toyed with technologies over millennia, and by focusing on the design path from automata to bots, Steenson forwards the idea that $\mathrm{Al}$ is not new. Al, she insisted, is an omnipresent phenomenon, an architectural mode of production based on the logic of architectural education and the history of design making. Her studies offer an infrastructural point of view that promotes the continuum or future agency of designing artificial intelligence as a series of active and interconnected elements roaming among all living things. She sees computing as the coupling of human knowledge $[\mathrm{HK}]$ and $\mathrm{Al}$, often altering design methods to further our creative logic. This perspective identifies the journey of numbers and patterns as an 
intellectual force, leading designers to view the mechanics of intelligence as part of a humanistic approach to innovation.

\section{ONE COGNITIVE PSYCHOLOGIST}

IV. The Learning and Forgetting Lab ${ }^{14}$ is a research-led experiment established by Robert Bjork's team on the subject of "desirable difficulties." 15 The team's scientific studies identified that learners benefit from tools like space and time. Among the team's highlights, they feature interleaving and spacing to infer their findings concerning memorization and retention.

1) Interleaving "occurs when a to-be-learned target item is initially presented and followed by different to-be-learned items, prior to the target's subsequent presentations. An interleaving schedule used during the presentation of a painting matched with the name of the artist has been shown to lead to better perfor-mance on later recognition tests when compared with a massing presentation schedule in which each painting and artist name was presented back-to-back, with one presentation immediately followed by the next presentation of a different painting by the same artist." 16

2) Spacing Effect "is the finding that information that is present-ed repeatedly over spaced intervals is learned much better than information that is repeated without intervals (i.e., massed presentation). This effect is one of the most robust results in all of cognitive psychology and has been shown to be effective over a large range of stimuli and retention intervals from nonsense sylla-bles to foreign language learning across many months." 17

Coupling cognition with space and time enhances human recognition and ensures the development of human's collective knowledge. The problem today is that we are told Al also learns through recognition and added data, yet, a biologist believes that what separates AI from all living organisms lies in the organic ability to hone and diversify knowledge over space and time. Under these purviews, designers will need to be less focused on pattern recognition, more focused on augmenting knowledge based on incremental experiences, and slow learning processes that will lead to calibrated ideas over extended periods of time.

\section{OUR APPROACH}

\section{TINKERING WITH NOTATIONS}

is a teaching approach configured to explain the foundations of design to an interdisciplinary cohort of students by introducing them to the use of machine and human logics that consider how intersperse systems become intertwined models to enhance the learning environment?

Coffee shops, maker-bars, and share work arenas seemingly reinforce the idea that learning occurs everywhere. There is a need, however, to identify places, where learning spheres support the allowance of time to solve problems meditatively and the use of equipment to critically improve and augment knowledge over time. It brought me to recognize that places like ateliers, workshops, and design studios underpin the type of learning environments that support the correlation between deep learning and machine logic.

Subsequently, in recent years my teaching methodology is exploring the deterministic behavior supported by digital receptors like phones, laptops, tablets, CNC, 3D printers, and a like, by looking for methods that would propel students to challenge their predictions and yield learning outputs that are experiential in their nature. This straight forward intention introduced a playful agenda in a collaborative dynamic workplace that would set the stage to heuristically forge a collective of players and to seize the school's fabrication lab in discovering how to control unpredictable outputs.

Those pursuits lead to recondition of the "as-is" understandings within studio context and to design assignments in correlation with accessibility to the equipment. Three goals were set forth for students and to initiate a new understanding of performativity. The first performance-based goal required students to personalize the manner in which any given tool could control the outcome of drawing grids. This task resulted in a series of drawing iterations stemming from productions and reproductions of combinatorial two \& three- dimensional work. It sprung the grounds for creative additives or extensions to an interface with the axis of pre-formatted machinery, such as drawing tables based on CNC technology. This action forged a new learning mandate, triggered by the realization that "an infinite series of interactive processes and decision-making opportunities could occur between what the machine was pre-formatted to do and what the human could intellectually, socially, and actively alter. Manifold events linking the physicality of making drawings using customized and adaptable jigs became a direct response for the production of a cyclical design process. In translation, all teams designed jigs, which needed extensive testing to ensure that they attach safely to the machine's tool head and that the machine could withstand the rigor of movement; speed and torque imposed by the new design condition-the repurposing of the tool head. Maintaining consistent results with various media types, dynamics of movement, and rigidity of the jig informed additional design decisions related to material strength, weight, and method of attachments. This also forwards attention to how the detailing of media receivers and the machine's tool head must be synchro-nized to work. After several design iterations, the customized jigs afforded us opportunities to test rapidly and prototype the per-formativity of the tools. Many of these jigs were reconfigurable and capable of additional rotations along specific $X Y Z$ axes and, as a result, these alternatives challenged our ability to control the behavior of the equipment. In time, these auxiliary axes augment-ed the prescriptive interpolations of non-linear directions, unveil-ing the manifold potentialities of the jig as an instrument and its latent potential to generate un-prescriptive outcomes." 18 




Figure 1,2. Material texture and directionality of tool path.

The second undertaking was initially based on a series of film viewings depicting the designing process of master chefs whose ability to discover and identify edible materials starts to engage the student in detecting opportunities to represent sensorial design processes from our environment. Here, beyond the joy of viewing farms, bioengineered food, and designing dishes to be served in high end restaurants, the sequential exercises encourage teams to engage in communicating ideas and providing revisions interactively, in realtime as a drawing documentary for how to format drawings that tell a narrative about meditative process.

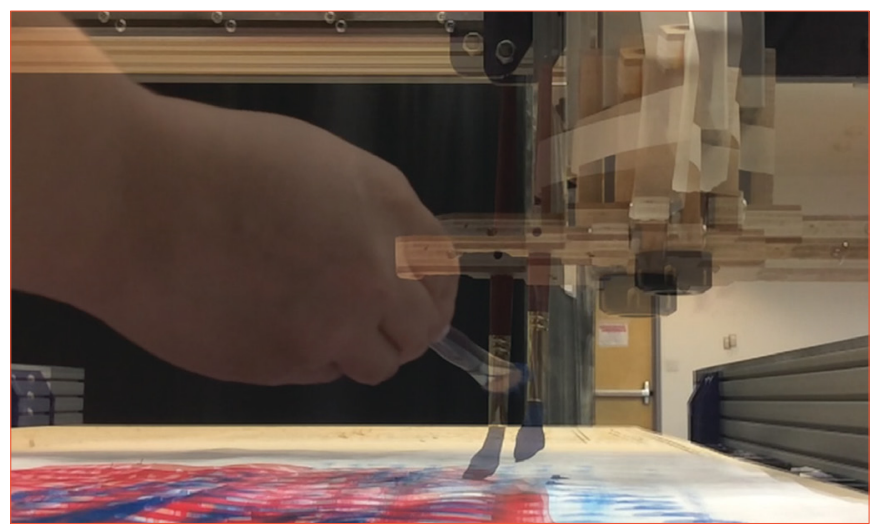

Figure 3. The digital hand; watercolor was intially resolved using an analog method.

The third and last goal which aimed to connect both exercises unreeled a discourse of the outcomes that clearly demonstrate how intervening in established sequences of operations introduces new potentials to divert the original behavior of the machine in ways that every printed iteration can be understood as an authentic original drawing as well as a new platform to depict meditative creative processes linking design thinking and making.

From a one-semester perspective, the production of these drawings renders a series of exercises that add to the re-understanding of design processes without the stigma of any specific design discipline. It focuses exclusively on the premise of communication andlogic inherent in designing processes. Lastly, students learned to identify

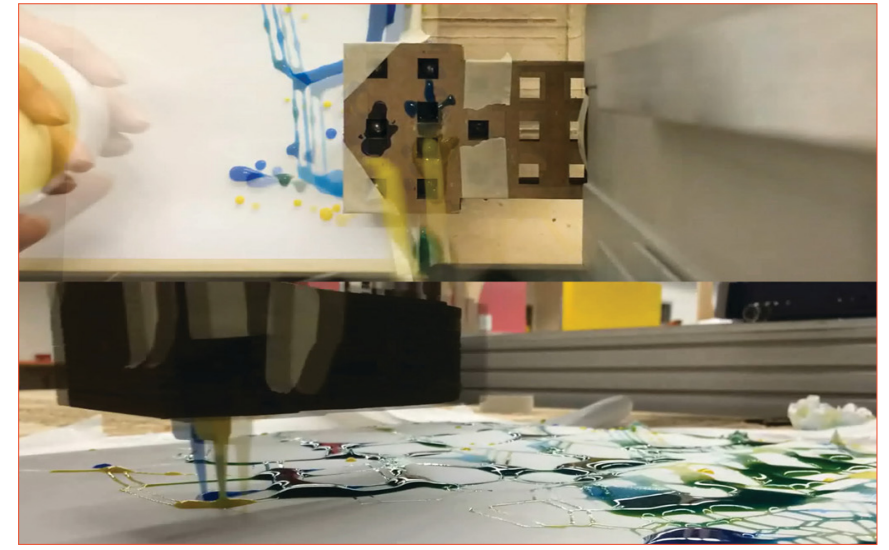

Figure 4. The Drip-jig with 2 color holder.

how to modify standardized computer-controlled equipment, engaging in digital-analog explorations that are not related to scripted codes. Each of the outcomes disrupted predetermined rules, noting that the efforts sought to embed prefabricated tools with new potential can produce infinitely flexible and dynamic outcomes, even when the instructions stem from a single G-code file.

\section{THE LABYRINTH OF MEMORIES}

is an Urban Culture and Urban Planning approach to teach co-dependent coefficients to organize and yield physical manifestations of our urbanity. The goal is to make sense of urban change in a classroom setting.

In contemplating Joshua Foer's message about the art and science of remembering everything; the secret, he says, is not intelligence, per say, it is a matter of learning to think in more memorable ways. This postulation comes from Robert Darton, who, in "The First Steps Toward a History of Reading," describes a switch from "intensive" to "extensive" reading that occurred as books began to proliferate. Until relatively recently, people read "intensively," says Darnton. "They had only a few books - the Bible, an almanac, a devotional work or two - and they read them over and over again, usually aloud and in groups, so that a narrow range of traditional litera-ture became deeply impressed on their consciousness." 19

Darnton has inspired my perspective to teach first-generation students the art of reading intensively rather than extensive exposure to data. I asked, how can complex urban theories become associations that expatiate modes for learning?

To answer this question, I was particularly interested in choreographing data and in limiting the amount of information, and historical range students are assigned per semester. The objective of my purview was to capture a student's focus and to stimulate a student's desire in developing the ability to process, sort and classify data in meaningful ways, using a similar methodology to how a musical instrument is taught or learned.

A readers list of selective work from the last one hundred years was shuffled as a lottery game between twenty-one students. Seven books from the disperse disciplines of philosophy, history, 
anthropology, urban design, policymaking, migration, and manufacturing economies ensure that seven teams of three students would read the ideas and theories of seven different authors even though not one student received the same articles (Fig5). The goal was to narrow the students with immediate reading responsibilities. The first assignment was simple, each student would take their personalized reading list and go through every article to highlight the words, people's names, and places that were new to them or that they did not fully understand. Followed by an assignment to define, identify, and map each one of these unrecognized arenas.

Figure 5. The readers list.

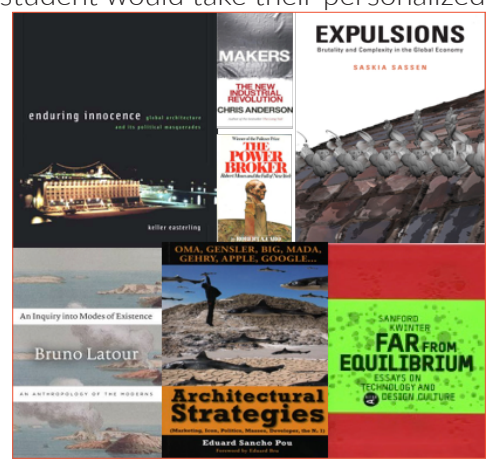

After these two tasks were accomplished, students had to re-read the articles to themselves and then aloud in front of their teammates. Soon after, they would be asked to extrapolate a discourse about common threads or disperse ideas within the authors. Aided by added information from contemporary podcasts, news journals, research papers, film documentaries, and other media outlets, they produced a personal summary and a group presentation. (Figure 7)

Figure 6. The Mapping of text.



During each presentation, the audience was asked to map common threads and note how the authors were studied; individually identifying the manufactured realities stemming from the team's presentation. From each presentation, the class uncover how dispersed disciplines forward similar, yet, invisible urban knowledge. More profound exercises ensued, and a dictionary of urban culture was forged with news podcast igniting verbal methods for mapping productions that established the grounds for a complex view about issues of urban culture challenging our contemporary believes about urbanity. (Figure 7) I have learned that working through the meaning of data using books, social media, computer graphics, CNC machines, laser cutters, and other available mediums forwards ideas and conflicts. This kind of project ought to take more than a semester and engage other critics in order to forward its depth and meaning. In the future, I would like to aim for more time to design thinking and learning seminars that thread the perception of design as the basis for a holistic education that encompasses our cultural systems of thinking and doing.
Perhaps a smaller readers list or fewer frames of perception?

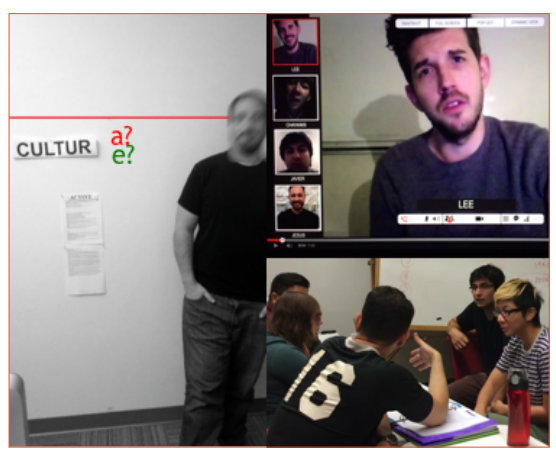

Figure 7. Reading out loud and the exchange of ideas.

\section{THE ART OF CONTEXT}

as framed by Eric Strain's design studio contemplates the fact that eighty years ago the idea of in-situ art, of land as the essential resource for creativity, and of a vision to reform the materiality and scale of the landscape gave birth to Land Art. This act of creation has both direct and indirect physical and philosophical implications to architecture. Few aspiring architects, however, have the opportunity to visit, contemplate, and reflect on land art and its contextual environment. As a source of creativity, Nevada's desert is home to Double Negative by Michael Heizer ${ }^{20}$ and Sun Tunnels by Nancy Holt, ${ }^{21}$ and I chose these installations to introduce students to the idea of site-specificity. The desert is the epicenter of a semester-long discovery about the environments we build in. The crux of a place has always dominated our learning praxis, and we have always wondered about this outlook, and the unrecognized opportunities identified through the diversity of territoriality and environment. Here, steeped with the understanding that location is critical to determine the polarities inherent in how schools of architecture build knowledge, my students are transported to camp a night near Double Negative, documenting what they experience via sketches, photographs, and material recordings to produce alternative means to explain the contrast of site specificities. The realities of the desert, its powerful winds, intense sunlight, cold nights, hot days, horizons, starry nights, and lack of human scale, are understood through contemplative experience and discovery, rather than abstracted theory or ideas without context, and the breadth of time spent within these realities allow for reflection on their implications. This experience transitions into a four-week-long exercise, revisiting and reflecting on their memory of the site, guided by the following questions: does the physical territoriality of place and identity matter in the production of innovation? How does data collection or 'radical surroundings' inform and form future praxises? Is the reasoning of future architects indoctrinated? How can they learn to selectively delete impertinent information stemming from both the praxises in academia and overcrowded volumes of data-banks?

Examples of their acts are as follows:

Contextual awareness and space were examined through a series of castings of the site and its surrounding topography, exploring both the man-made cuts into the landscape as well as those created by the forces of erosion. 

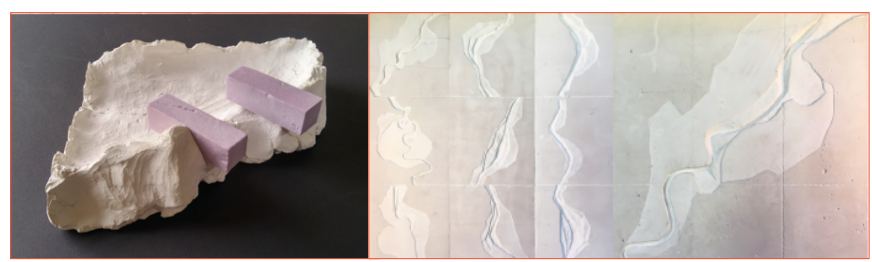

Figure 8. Casting.

The primary focus was the exploration of space and the multiple feelings it could elicit. This is created by subtraction rather than addition, a volumetric cavity is removed from the mesa in the same manner as Michael Heizer, open to the sky above. In the vastness of the desert mesa, distractions are minimized, and attention is focused through space and volume, evoking an aura of tranquility and contemplativeness.

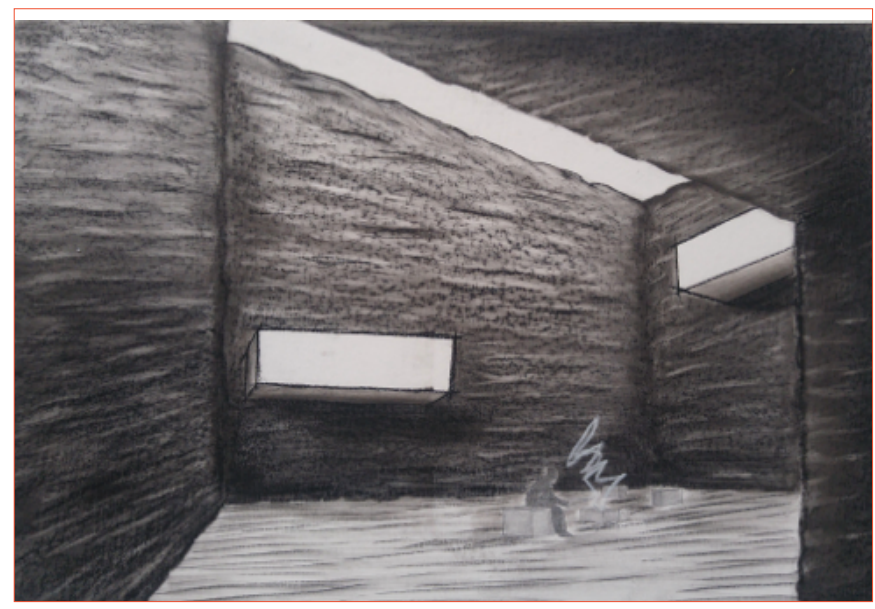

Figure 9. Exploring media type in the articulation of the void.


Figure 10. Expressive sketching connecting landscape with ideas.

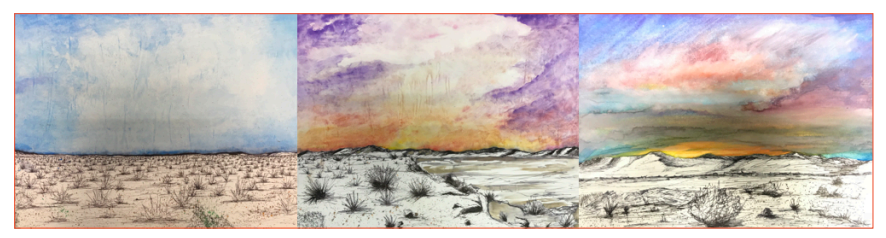

Figure 10. Sketching empitness.

Contemplation of that great vastness of the site led to an interrogation of how humans connect with nature. Through the psychological concept of 'prospect and refuge,' the expansive desert horizon is replaced with various framed views experienced from tunnels cut into the mesa. The earth, acting as the frame and focusing one's gaze, also provides a sense of refuge, intimacy, and shelter in a wide-open space.

However, the belief that any man-made change, addition or subtraction, to the site would take away the experiential qualities and original intent of the land art piece cut already into the mesa, the decision to build nothing - and what that means to a designer of built works - was also explored. 


\section{Notes}

1 In 2003, No. 19/Architectural as Conceptual Art? Blurring Disciplinary Boundaries, published an article by T. Love, explaining the propagation of the Kit of Parts as a nine-square grid educational exercise. A press released [No. 134] published by MOMA, November 12, 1971, pronounced Cooper Union's as the leader on the subject of the education of an architect. The announcement of the exhibition also highlighted seven years of teaching at Cooper Union, where the Kit of Parts and the nine-square grid exercises of the times.

http://www.harvarddesignmagazine.org/issues/19/kit-of-parts-conceptualism-abstracting-architecture-in-the-american-academy

https://www.moma.org/documents/moma_catalogue_2648_300298971.pdf

2 In 2009 WIRED magazine published an article Blobitecture and Parametrics by Bruce Sterling that questioned the value of blobitecture once drawing-software would be cheap and easy for everyone to access and this is, of course, is an exciting subject to reflect on as our educational institutions today start to view mix and match realities in the spatial definitions of architecture .

From wired magazine's website: https://www.wired.com/2009/07/blobitecture-and-parametrics/

From Lecture at SCI-Arc 2018 Lecture Series https://www.youtube.com/ watch?v=-p4DV80xrm8

3 Aravena, Alejandro and Andres Lacobelli from Elemental developed the Incremental Housing Project as an academic initiative, turned it into a "do it yourself type of practice." It originates from the desire to stimulate social conscious and to configure a type of Participatory Design Manual that ultimately yielded a Pritzker price.

The Book: Elemental by Aravena, Alejandro and Andres Lacobelli was published by Hatje Cantz Verlag, 2013

View books briefing: https://www.hatjecantz.de/elemental-5360-1.html

4 The Susskind's [son and father team Susskind, Richard, and Daniel Susskind] examined the ways technologies are not copying human capacity but rather augment our rational and living progress. They wrote a book: The Future of the Professions: How Technology Will Transform The Work of Human Experts. Oxford: Oxford University Press, 2015

The idea is to that we should focus on our modes of economic innovation rather than work.

https://www.youtube.com/watch?v=Yzi6kkfXqt8

5 O’Donnell, Kathleen wrote an article for aia.org titled Embracing artificial intelligence in architecture. Her argument focuses on our need to go with the flow and embrace the path toward machine intelligence. This idea simplifies our academic goals and forwards the road for us to imagine a future with more time to think. aia.org. March 2, 2018. https://www.aia.org/ articles/178511-embracing-artificial-intelligence-in-archit:46

6 Vincent Van Gogh's best work focused on the basic needs of human survival. Reflections of how we rest, nourish, cleanse, and think, are part of his paintings and the portrayal of the outcome rural populations suffered in striving to achieve these goals. The letter to Emile Bernard is part of the vangoghmuseum.nl collection, and we chose this extract as telling of the insight Van Gogh perceives as soon as he left Paris. The text is also unveiling of the argument others made about Vincent's meditative work. We suggest a visit to David Hockney's admiration for the Dutch master by Richard Holledge.

https://gulfnews.com/entertainment/arts-culture/a-walk-in-the-woods-withvincent-van-gogh-1.62464866

7 The Radical Pedagogies Project [RPP], per our studies and Colomina's explanation, is a living system that aids us to reflect on the recent history for educating future architects. Additional information is now on Youtube: https://www.youtube.com/watch? $\mathrm{v}=\mathrm{qPog} 2 \mathrm{YVNVnw}$; The Venice architectural biennale awards: https://www.labiennale.org/en/architecture/2014/premi; and Radical Pedagogies Website: https://radical-pedagogies. com

8 On the topic of Learning Network and RPP, a thirty-two-page article in Volume \#45 titled LEARNING, Colomina and collogues explained the veracity of reconstructing an architectural education http://volumeproject.org/the-radical-pedagogies-project/

9 From Graham's Foundation site we learned that RPP received funding to develop the first book on their research about pedagogy in architecture: http://www.grahamfoundation.org/grantees/5949-radical-pedagogies

10 David Basulto: https://www.archdaily.com/518281/venice-biennale-2014-radical-pedagogies-exhibit-design-by-amunategui-valdes-architects

11 Beyond the book by Carpo, Mario. The Second Digital Age: Design Beyond Intelligence. Cambridge: MIT Press, 2017, we looked at a series of lectures and conversation available on academic sites that highlight the spectrum of Carpo's research work regarding our technologies through times. We have studied his point of view from multiple of perspective. The studies helped us grasp a broader definition of learning in the age of digitals. It $t$ aid us in developing our approach to slow learning and time to think in the design studio.

12 Metropolis Magazine Q/A: Mario Carpo on Architecture's Digital Past and Present. https://www.metropolismag.com/ideas/design-education/ qa-mario-carpo-on-architectures-digital-past-and-present/

13 Architectural Intelligence: How Designers and Architects Created the Digital Landscape published by MIT Press in 2017, is a book that stirred ideas about multiple actions to educate future designers. The book helps to rethink the value of intelligence from the trajectory of ancient to contemporary thinkers. Steenson's website http://www.girlwonder.com reflects on j176: media fluency in the digital age and addresses the idea that the course will try to "slow-down" to "examine" digital media. We see this intent as an approach to what we are learning to identify as "time to think" and to defend slow teaching.

14 At UCLA Bjork's Learning and Forgetting Lab https://bjorklab. psych.ucla.edu/research/ deals with cognitive psychology and looks to enhance educational practices by testing the timing feedback of memory and performance.

15 In 2008 Kornell and Bjork published a paper in the Association of Psychological Science title Learning Concepts and Categories. The article described Desirable Difficulties as a learning mechanism dependent on the duration process an individual necessitates to learn. In other words, memory happens in time, and this is necessary for all kinds of approaches to retain information, which returned us to the idea that learning is a craft.

16 For further explanations about the concept of Interleaving visit: https://bjorklab.psych.ucla.edu/research/\#interleaving

17 For further explanations about the concept of Spacing visit: https://bjorklab.psych.ucla.edu/research/\#spacing

18 The students work as explained at the 2017 National Conference for Beginning Design Students and its proceedings under the titled CNC Mediated Representation by Yeshayahu, Vermillion, and Solano.

19 In the book Moonwalking with Einstein: The Art and Science of Remembering Everything, Joshua Foer recounts a yearlong experiment on memory. He details the outcome of his achievements and recollections 
19 In the book Moonwalking with Einstein: The Art and Science of Remembering Everything, Joshua Foer recounts a yearlong experiment on memory. He details the outcome of his achievements and recollections regarding the exercises to retain information and to recall data for future references.

20 As the first and perhaps most symbolic approach to Land Art, Double Negative by Michael Heizer, is also an architectural experiment. Consider a sculpture, the carving of the earth does not fit inside a museum, but it is own by MOCA. It is an ephemeral work of art on its way to extinction. In so many ways, this work is an environmental explanation of time. http://doublenegative.tarasen.net/double-negative

21 Sun Tunnels by Nancy Holt, like Double Negative, speaks of time as a lapse of duration and serves to focus the immensity of scale and site from the perspective of the sunlight and time. It creates a meditative approach to reflect on the point of view through the time of earth's ration, speed, and days in a year. https://www.google.com/search?client=safa-

ri\&rls=en\&q=21.+Sun+Tunnels + by + Nancy + Holt \&ie $=$ UTF- $8 \& o e=U T F-8$ 